\title{
Kaugaliang Pilipino at ang kabataan ngayon tungo sa programang edukasyon sa pagpapakatao
}

Mansanadez, Evangeline $\$
Jose Rizal Memorial State University - Tampilisan Campus, Philippines (evangelinemansanadez@gmail.com)

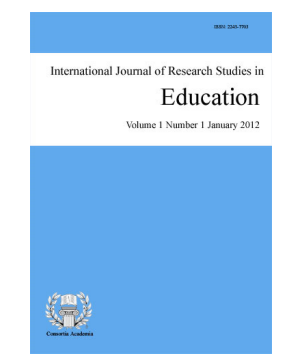

Received: 30 March 2021

Revised: 5 May 2021

Available Online: 10 June 2021

\section{Abstract}

This study aimed to determine the valuing extent of the Filipino traits by the Junior High School students of Tampilisan District. It made use of a descriptive-quantitative survey method of research, employing a researcher-made questionnaire-checklist in gathering the necessary data of the study, involving the 390 students as respondents of the study. Data were analyzed using the weighted mean and Independent T-test. Findings revealed that the Filipino trait on the love of God such as "respect for the Holy Bible or Qur' an and respect for the church" were still valued by the respondents according to sex. In terms of the love of people were "thoughtful to parents and siblings; respectful to the elders; and having a close relationship to the family". On the love of country were "respect for the national heroes; proud of being a Filipino; respect for the national symbols such as the national anthem, flag and others; patronizing the national language by using it at home or in school; and attending the flag raising ceremony". On the love of nature was "cleaning the environment". The extent of valuing by both the female and male respondents on the love of God, love of people and love of nature is "often" while on the love of country is "always" for the females and "often" for males. There is no significant difference on the valuing of the love of God, love of nature and love of country but a significant difference existed in terms of the love of people.

Keywords: Filipino traits; love of God; love of people; love of country; love of nature 


\section{Kaugaliang Pilipino at ang kabataan ngayon tungo sa programang edukasyon sa pagpapakatao}

\section{Introduksyon}

Ang Pilipinas na binansagang "Perlas ng Silangan" ay isa sa mga bansang biniyayaan ng Poong Maykapal sa hindi mabibilang na likas na yaman, kahanga-hangang kapaligirang nakapanghalina at mamamayang may taglay na magagandang asal at pag-uugali. Ang pagiging magiliw, magalang, matulungin, masipag, relihiyoso, at iba pang kaaya-ayang katangian ng mga Pilipino, maliban pa sa mga magagandang tanawin sa bansa, ang nagpapakilala at nagpapatanyag nito sa buong mundo. Bunga ng pagkakaroon ng mayamang kultura ng mga Pilipino ang dahilan kaya kinagigiliwan ang mga ito ng mga dayuhan at samot-saring turista ang nahihikayat na magliwaliw rito. Ngunit, ayon kay Ruto (2017), nawawala na ang mga mabubuting asal na dapat sana ay ipagpatuloy pa at panatilihin. Para sa kanya, halos lahat ng kabataan ngayon ay wala nang galang at respeto sa mga nakakatanda at hindi na nila binibigyang-halaga ang mga kaugaliang ipinamana at itinuro sa kanila.

Ipinabatid din ng The Asian Parent Philippines (n.d) ang kanilang naobserbahan na iba na raw ang mga bata ngayon - makabago na raw. Iminungkahi nitong hindi pa rin dapat kalimutan ang mabuting asal at kaugaliang Pilipino na kinalakihan ng kanilang mga magulang at nararapat na ipakita, ituro at isabuhay sa araw-araw ng mga magulang para maging mabuting impluwensiya sa kanilang mga anak dahil ito raw kasi ang gagabay sa kanila at magagamit nila habambuhay sa pakikitungo at pakikisalamuha sa iba't ibang tao. Para sa kanila, ang mga mabuting asal at kaugaliang Pilipino na dapat ituro sa mga bata ay ang mga sumusunod: magalang na pananalita, paghingi ng permiso, pangangamusta, huwag manghimasok, wastong ugali sa hapag-kainan, huwag maging mapanghusga, pagrespeto sa personal space, pagiging masinop, paglinis ng kapaligiran, pagkilala sa mga tao sa kanilang paligid, at pagmamahal sa bansa.

Samantala, ipinahayag ni Cruz (2015) sa kanyang blog ang kanyang kalungkutan na unti-unti nang nawawala ang mga magagandang katangian ng mga Pilipino na sana ay mabago at hindi na maisalin pa sa mga susunod na henerasyon dahil batay sa kanyang nakikita sa kabataan maging sa mga matatanda ngayon ay abala na sa paglalaro ng online games, xbox, tablets, pati facebook at instagram sa halip na nakikipag-socialize sa iba. Para sa kanya, hindi naman daw masama ang maglibang ngunit sabi nga sa kawikaan, "lahat ng sobra ay masama”. Dagdag pa niya, kahit ang ibang mga magulang din ay nag-iba na rin daw ang pananaw sa pagdaan ng panahon. Minsan nalang daw lumabas ng kwarto kaya kahit normal na family gathering ay nawawala na. Magkakasama nga raw sa iisang bubong ngunit wala nang oras para mag-usap. Idinagdag pa niya na hindi naman daw nawala sa ibang Pilipino ang mga magagandang katangian nito, ngunit nang dahil sa modernisasyon ay nakakalimutan na ito.

Sa ibang dako naman, taliwas sa naunang pahayag ang kinalabasan ng pag-aaral ni Suazo (2019) na naghahayag na ang katangian ng mga milenyal na nahawaan ng Facebook ay ang humugis sa kanilang pakikipag-ugnayan at pakikisalamuha sa tunay na mundong kanilang ginagalawan. Ang kanilang katangiang taglay ay katulad pa rin ng tradisyunal na katangiang mayroon ang mga intelektwal, kaya lang, naiiba ang kanilang kaparaanan ng paggamit nito.

Kaugnay nito, lumabas sa pag-aaral ni Obille (2018) na hinangaan ng mga respondente ang iba-ibang pinagkukunan ng impormasyon maging ito ay online at offline, may mga social media accounts at nakikitang may gamit ang bawat isa depende lamang sa kanilang pangangailangan at konteksto, palaging online sa lahat ng panahon at palaging umaasa sa impormasyon. Ang kaugaliang "hiya", "gaya-gaya" at "pagkakaibigan" ay ipinakita sa kanilang ginamit na SNS, kung anong impormasyon ang maaaring ibahagi at kung paano sila makipag-ugnayan sa kanilang mga kaibigang nasa online at offline.

Sa blogspot naman ni Alcober (2016), sinabi niyang sa panahon ngayon, kapansin-pansin ang unti-unting 
pagkalimot sa mga kaugaliang Pilipino ng mga kabataan tulad ng pagmamano at paggamit ng "po" at "opo" at halos wala nang pakialam sa pagrespeto sa mga nakatatanda. Para sa kanya, wala mang pormal na edukasyon noon, hindi raw ito naging hadlang upang makilala ang mga Pilipino sa pagiging mahusay sa pakikipagkapwa-tao. Isa pa rin daw sa mga kinalimutan ng mga kabataan ngayon ay ang mga larong pinoy, gaya ng luksong baka, bahay-bahayan at iba pa dahil sa impluwensya ng makabagong teknolohiya, kaya, puro mga computer games ang nilalaro at halos wala nang oras para makihalubilo sa ibang kabataan sa labas. Iminungkahi niyang sana ay isipin na pahalagahan ng bawat isa ang magandang katangian ng mga Pilipino. Kaugnay sa pahayag na ito, ang dahilan ng pagkalimot sa mga kultura at tradisyong Pilipino ay ang makabagong teknolohiya at "colonial mentality" (Credo et al., 2019; Rosela, 2014).

Ngunit, ayon pa sa ibinahagi ng MyInfo Basket.com (2020), ang mga Pilipino ay magalang at mapitagan na kahit sa modernong panahon, marami pa rin ang nagpapakita ng halaga sa kani-kanilang mga pamantayan sa buhay at mga tradisyon. Kaya, iminungkahi nitong nararapat lamang na ipagpatuloy ng bawat isa ang magagandang kaugaliang tulad ng paggalang sa mga nakatatanda at iba pa sa pamamagitan ng paggamit ng "po at opo"; pagiging magiliw sa panauhin at pagiging relihiyoso (del Castillo et. al, 2021); paggamit ng sariling wika bilang pagpapatunay at pagpipreserba ng pambansang pagkakakilanlan upang makamtan ang pagkakaisa at pambansang kaunlaran; at pagpapamalas ng mga Pilipino sa kakanyahan ng nasyonalismo sa araw-araw na pakikipamuhay.

\section{Teoretikal na balangkas ng pag-aaral}

Ang pag-aaral na ito ay nababatay sa Social Learning Theory ni Bandura (1977) na binanggit ni McLeod (2016). Ayon sa teoryang ito, ang tao ay natututo mula sa kanyang kapwa sa pamamagitan ng pagmamasid, pagsunod, at pagkahubog mula rito. Ang teoryang ito ay tinatawag ding tulay sa pagitan ng Behaviorist at Cognitive Learning Theories dahil pareho itong sumasaklaw sa pansin, memorya, at motibasyon.

Ang teoryang ito ay nagsasabing ang tao ay natututo sa pamamagitan ng pagmamasid sa gawi ng iba at ang kanyang pag-uugali ang kinalabasan ng mga ito. Kadalasan, ang ugali ng tao ay natutuhan sa pamamagitan ng pagmamatyag at pagmomolde mula sa mga naoobserbahan sa iba. Ang ideya ay nabubuo kung paano ito gaganapin at hindi maglaon, ang kaalamang ito ay nagsisilbing gabay para sa paggawa. Ang Social Learning Theory ni Bandura ay nagpapaliwanag na ang pag-uugali ng tao ay nahuhubog dahil sa patuloy na interaksyon nito gamit ang kanyang kaisipan, asal at impluwensyang pangkapaligiran.

Naniniwala rin si Bandura sa reciprocal determinism na ang mundo ang siyang dahilan ng pagkahubog ng bawat tao at ang kapaligiran ang siyang dahilan ng pag-uugali ng bawat isa. Sa pag-aaral ni Bandura tungkol sa Adolescent Agression, natuklasan niyang ang pag-uugali ay dahil sa kapaligiran. Sa kalaunan, isinaalang-alang nito ang personalidad bilang interaksyon sa tatlong komponent: Ang kapaligiran, kaugalian at ang proseso ng kaniya-kaniyang kaisipan.

\subsection{Legal na balangkas ng pag-aaral}

Isa pang pinagbabatayan ng pag-aaral na ito ay ang DepEd Order No. 31 s. 2012 ayon kay Llego (2019) na pinamagatang Policy Guidelines on the Implementation of Grades 1 to 10 of the $\mathrm{K}$ to 12 Basic Education Program (BEP) na nagkabisa sa Taong Panuruan 2012-2013 na nagtatakda ng mga detalye ng ilalaang oras sa bawat lawak ng pagkatuto sa lahat na baitang. Itinakda rito na ang Edukasyon sa Pagpapakatao (EsP), bilang isang lawak ng pagkatuto, ay ituturo sa loob ng tatlumpung (30) minuto araw-araw o isandaan at limampung (150) minuto bawat linggo sa elementarya at dalawang (2) oras naman bawat linggo o isang (1) oras bawat araw sa loob ng dalawang (2) araw bawat linggo para sa Junior High School (JHS).

Ang dalawang (2) oras bawat linggo o isang (1) oras bawat araw na inilaan sa loob ng dalawang (2) araw bawat linggo ay dapat sulitin sa pagtuturo sa mga kakayahang nararapat linangin sa mga alituntunin ng pagkatuto na natatadhana sa Gabay ng Kurikulum mula Baitang 7 hanggang 10. 


\subsection{Layunin ng pag-aaral}

Ang pag-aaral na ito ay isang pagtatangka upang malaman ang kalagayan ng kaugaliang Pilipino at ang kabataan ngayon tungo sa programa sa edukasyon sa pagpapakatao.

Nilalayon nitong masagot ang mga sumusunod:

$>\quad$ Ang dalas ng pagpapahalaga ng mga respondente sa kaugaliang Pilipino kung uuriin sila ayon sa kasarian

$>\quad$ Ang kaibahan sa pagpapahalaga ng mga respondente sa kaugaliang Pilipino ayon sa kasarian

$>\quad$ Ang programang maaaring isasagawa na maging batayan sa edukasyon sa pagpapakatao.

\section{Pamamaraan ng pananaliksik}

Ang pag-aaral na ito ay gumamit ng pamamaraang deskriptiv-kwantitativ na pananaliksik na isinagawa sa pamamagitan ng pagsarbey gamit ang kwestyoneyr-tseklist o talatanungan na binuo mismo ng mananaliksik gamit ang 30\% ng 1,303 ng mga mag-aaral ng Tampilisan National High School mula Grade VII hanggang Grade $\mathrm{X}$ bilang respondente ng pag-aaral na isinaalang-alang sa pamamagitan ng random sampling na teknik.

Ang instrumento ng pananaliksik ay pinagtibay ng tatlong (3) eksperto sa larangan ng pananaliksik na may digring doktor ng edukasyon. Upang lalo pang mapatunayan ang instrumento, isinagawa ang reliability test gamit ang 15 mag-aaral na hindi kabilang sa pag-aaral. Pagkatapos masagot ang talatanungan, ang resulta ng pagsubok ay sinuri gamit ang Cronbach's alpha test at lumabas na may valyung 0.70 na nagpapahiwatig na may mataas na lebel ng internal consistency ng sukatang ginamit ng pag-aaral.

Ang mga sumusunod ay ang batayan ng pagmamarka:

\begin{tabular}{ccc}
\hline Batayan ng Pagmamarka & Kowd & Deskripsyon: \\
\hline $3.25-4.00$ & Palagi & Pinapahalagahan sa lahat ng panahon \\
$2.50-3.24$ & Madalas & Pinapahalagahan sa halos lahat ng panahon \\
$1.75-2.49$ & Minsan & May panahong ito ay pinapahalagahan at mayroon ding hindi \\
$1.00-1.74$ & Bihira & Hindi masyadong pinapahalagahan \\
\hline
\end{tabular}

Bago ipinamahagi ang instrumento ng pananaliksik sa mga respondente ng pag-aaral, humingi muna ng pahintulot ang mananaliksik sa superbisor ng distrito at prinsipal upang makapangalap ng mga datos. Ang mga datos na nakalap ay sinuri gamit ang weighted mean at $t$-test.

\section{Kinalabasan ng pag-aaral at pagtalakay}

\subsection{Dalas ng pagpapahalaga sa kaugaliang Pilipino}

Maka-Diyos - Ang talahanayan 1 ay naglalahad sa mga datos ukol sa dalas ng pagpapahalaga ng mga mag-aaral ayon sa kasarian sa kaugaliang maka-Diyos. Ayon sa resulta, ang palaging pinapahalagahan ng mga respondente ayon sa kasarian ay ang pangwalong aytem "Iginagalang ang mga banal na gamit tulad ng bibliya $\mathrm{o}$ Koran at mga banal na lugar tulad ng simbahan" na may weighted mean na 3.39 para sa mga babae at 3.27 naman sa mga lalaki. Ibig sabihin, ang kapwa pinapahalagahan lamang ng mga mag-aaral ay ang mga kagamitang nauukol sa pananampalataya. Samantala, ang kabuuang weighted mean na 2.80 ay nagpapakita na ang dalas ng pagpapahalaga ng mga respondente ayon sa kasarian ay madalas. Ibig sabihin, pinapahalagahan nila ang pagiging maka-Diyos sa halos lahat ng panahon.

Ayon kay Cordero (2019) sa kanyang ginawang pag-aaral, sa modernong panahon ngayon, ang pagbaba ng moralidad sa kabataan ay higit na nalalantad. Tatlong pangunahing dahilan ang kanyang nakikita: una, ang medya, 
panggigipit o impluwensya ng mga kasamahan, at kakulangan sa kalinga at suporta ng pamilya. Kaya, iminungkahi niya na kinakailangan ang agarang pagtuturo ng ebanghelyo sa mga kabataan. Idinagdag niyang ito ngayon ang gawain ng kontekstwal na teolohiya, isang uri ng pagtuturo hinggil sa pananampalataya sa Poong Maykapal na angkop sa konteksto ng kultura ng kabataan. Natuklasan naman ni Kahambing (2019) na ang mga estudyante sa Pilipinas na nagpakadalubhasa sa kani-kanilang kurso ay hantad sa konteksto ng kulturang Pilipino. Ang kaugaliang Pilipino ay kadalasang nagkahugis sa pamamagitan ng matas na antas ng perspektibo ng relihiyong Katoliko na isinalin tungo sa moral na panuntunan at nang lumaon ay hinubog tungo sa Pilipinong kapisanan at kultura.

\section{Talahanayan 1}

Dalas ng pagpapahalaga ng mga respondente sa kaugaliang maka-Diyos ayon sa kasarian

\begin{tabular}{|c|c|c|c|c|c|c|}
\hline \multirow{2}{*}{ Maka-Diyos } & \multicolumn{2}{|c|}{ Babae } & \multicolumn{2}{|c|}{ Lalaki } & \multicolumn{2}{|c|}{ Kabuuan } \\
\hline & WM & Des & WM & Des & WM & Des \\
\hline 1. Nagdadasal tuwing alas-tres ng hapon & 2.67 & $\mathrm{Ma}$ & 2.49 & $\mathrm{Mi}$ & 2.58 & $\mathrm{Ma}$ \\
\hline 2. Nagdadasal bago matulog & 2.96 & $\mathrm{Ma}$ & 2.90 & $\mathrm{Ma}$ & 2.93 & $\mathrm{Ma}$ \\
\hline 3. Nagdadasal bago kumain & 2.63 & $\mathrm{Ma}$ & 2.65 & $\mathrm{Ma}$ & 2.64 & $\mathrm{Ma}$ \\
\hline 4. Nagdadasal pagkagising & 2.42 & $\mathrm{Mi}$ & 2.50 & $\mathrm{Ma}$ & 2.46 & $\mathrm{Mi}$ \\
\hline $\begin{array}{l}\text { 5. Nagsisimba tuwing Linggo o sabado o ibang araw na } \\
\text { itinakda na pagsamba }\end{array}$ & 2.83 & $\mathrm{Ma}$ & 2.71 & $\mathrm{Ma}$ & 2.77 & $\mathrm{Ma}$ \\
\hline 6. Hindi tamad magsimba & 2.82 & $\mathrm{Ma}$ & 2.69 & $\mathrm{Ma}$ & 2.76 & Ma \\
\hline 7. Aktibo sa mga gawaing pansimbahan & 2.76 & $\mathrm{Ma}$ & 2.78 & $\mathrm{Ma}$ & 2.77 & $\mathrm{Ma}$ \\
\hline $\begin{array}{l}\text { 8. Iginagalang ang mga banal na gamit tulad ng bibliya o } \\
\text { Koran at mga banal na lugar tulad ng simbahan }\end{array}$ & 3.37 & $\mathrm{P}$ & 3.27 & $\mathrm{P}$ & 3.32 & $\mathrm{P}$ \\
\hline $\begin{array}{l}\text { 9. Iginagalang ang uri ng pananampalataya o relihiyon ng } \\
\text { kapwa }\end{array}$ & 3.39 & $\mathrm{P}$ & 3.21 & $\mathrm{Ma}$ & 3.3 & $\mathrm{Ma}$ \\
\hline 10. Hindi mahilig gumamit ng mga masasamang salita & 2.77 & $\mathrm{Ma}$ & 2.65 & $\mathrm{Ma}$ & 2.71 & $\mathrm{Ma}$ \\
\hline 11. Hindi nagsisinungaling & 2.52 & $\mathrm{Ma}$ & 2.49 & $\mathrm{Mi}$ & 2.51 & $\mathrm{Ma}$ \\
\hline 12. Hindi nagnanakaw & 2.74 & $\mathrm{Ma}$ & 2.91 & $\mathrm{Ma}$ & 2.83 & $\mathrm{Ma}$ \\
\hline Kabuuang Mean & 2.82 & $\mathrm{Ma}$ & 2.77 & $\mathrm{Ma}$ & 2.80 & $\mathrm{Ma}$ \\
\hline
\end{tabular}

Maka-tao - Ang talahanayan 2 ay naglalahad ng mga datos ukol sa dalas ng pagpapahalaga ng mga respondente ayon sa kasarian sa kaugaliang maka-tao. Batay sa mga datos, ang mga aytem na "Maalalahanin sa mga magulang at sa kapwa", "Magalang sa mga nakatatanda at sa kapwa" na kapwa may weighted mean na 3.46, at "Malapit sa pamilya", 3.43 ay "palagi" ang dalas ng pagpapahalaga ng mga respondente ayon sa kasarian. Ito ay nagpapakita ng pagiging malapit at mapagmahal ng mga respondente sa kani-kanilang pamilya. Ibig sabihin, nananatili pa rin sa puso at isipan ng kabataang Pilipino ang ugaling "close family ties".

Sa pagkakaroon ng kabuuang weighted mean na 3.08, ang dalas ng pagpapahalaga ng mga respondente sa kaugaliang maka-tao ayon sa kasarian ay madalas. Ibig sabihin, pinapahalagahan nila ang pagiging maka-tao sa halos lahat ng panahon. Inihayag ni Jumawan (2019) ang kinalabasan ng kanyang pag-aaral na ang pamilya, respeto, at katapatan ay itinuring na pinakamahalaga para sa kanyang mga kalahok na positibong maiugnay sa "core values" ng mga Pilipino tulad ng pakikipagkapwa, pakiramdam, at kagandahang loob. Idinagdag niyang, batay sa nakikita sa personal na katangian ng mga respondente, ang diwa ng katauhan ng mga Pilipino ay nararapat na masasalamin bunga ng pagkakaroon ng Edukasyon sa Pagpapakatao bilang "social capital" para sa pagsulong ng kabuhayan ng nakararami at pulitikal na kaunlaran dito sa Pilipinas.

Ayon naman kay Reyes (2015) batay pa rin sa kanyang pag-aaral na ang kaugaliang Pilipinong "loob" at "kapwa" ay nagsilbing haligi ng espesyal na koleksyon ng kaugaliang kagandahang-loob, utang-na-loob, pakiramdam, hiya at lakas-ng-loob/bahala na hindi naman ugaling makasarili tulad ng kadalasang mga pangunahing pag-uugali ng kanluraning tradisyong kahinahunan, hustisya, pagtitimpi, at tibay ng loob; subalit ang lahat ay nakadirekta tungo sa pagpapanatili at pagpapatibay sa makataong ugnayan. Samantala, natuklasan naman nina Saito et al. (2010) ang sampung pag-uugaling madalas nilang nakikita sa mga Pilipino tulad ng pakikisama, hiya, utang na loob, pagkamalapit sa pamilya, bahala na, amor propio, bayanihan, pagkamagiliw sa panauhin, ningas-kugon, at paggalang sa mga nakatatanda. Si Chico (2012) sa kanyang pananaliksik, nabatid niya ang mga pangunahing kaugalian ng mga kabataang kalahok ng Pinoy Big Brother tulad ng lambing, 
Mansanadez, E.

pakikisama o pakikipagkapwa-tao, sikap at tiyaga at pagsasabi ng "po" at "opo" o paggalang.

\section{Talahanayan 2}

Dalas ng pagpapahalaga ng mga respondente sa kaugaliang maka-tao ayon sa kasarian

\begin{tabular}{|c|c|c|c|c|c|c|}
\hline \multirow{2}{*}{ Maka-tao } & \multicolumn{2}{|c|}{ Babae } & \multicolumn{2}{|c|}{ Lalaki } & \multicolumn{2}{|c|}{ Kabuuan } \\
\hline & WM & Des & WM & Des & WM & Des \\
\hline 1. Pagmamano o paghalik ng kamay sa nakakatanda & 3.25 & $\mathrm{P}$ & 2.93 & $\mathrm{Ma}$ & 3.09 & $\mathrm{Ma}$ \\
\hline 2. Maalalahanin sa mga magulang at sa kapwa & 3.54 & $\mathrm{P}$ & 3.37 & $\mathrm{P}$ & 3.46 & $\mathrm{P}$ \\
\hline 3. Magalang sa mga nakakatanda at sa kapwa & 3.53 & $\mathrm{P}$ & 3.32 & $\mathrm{P}$ & 3.46 & $\mathrm{P}$ \\
\hline 4. Matapat sa lahat ng Gawain & 3.07 & $\mathrm{Ma}$ & 2.95 & $\mathrm{Ma}$ & 3.01 & $\mathrm{Ma}$ \\
\hline 5. Magiliw sa pagtanggap ng panauhin & 3.04 & $\mathrm{Ma}$ & 2.90 & $\mathrm{Ma}$ & 2.97 & $\mathrm{Ma}$ \\
\hline $\begin{array}{l}\text { 6. Hindi mahilig sa mañana habit o sa ugaling "mamaya } \\
\text { na" }\end{array}$ & 2.64 & $\mathrm{Ma}$ & 2.62 & $\mathrm{Ma}$ & 2.63 & $\mathrm{Ma}$ \\
\hline 7. Matulungin sa kapwa & 2.97 & $\mathrm{Ma}$ & 2.95 & $\mathrm{Ma}$ & 2.96 & $\mathrm{Ma}$ \\
\hline 8. Hindi Ningas-kugon hindi mabuti lang sa simula & 2.68 & $\mathrm{Ma}$ & 2.63 & $\mathrm{Ma}$ & 2.66 & $\mathrm{Ma}$ \\
\hline 9. Malapit sa pamilya & 3.48 & $\mathrm{P}$ & 3.38 & $\mathrm{P}$ & 3.43 & $\mathrm{P}$ \\
\hline 10. Marunong tumanggap ng pagkatalo & 3.31 & $\mathrm{P}$ & 3.10 & $\mathrm{Ma}$ & 3.21 & $\mathrm{Ma}$ \\
\hline 11. Marunong makasama & 3.29 & $\mathrm{P}$ & 3.17 & $\mathrm{Ma}$ & 3.23 & $\mathrm{Ma}$ \\
\hline $\begin{array}{l}\text { 12. Hindi mahilig magpasikat, magpabongga at } \\
\text { magpaporma }\end{array}$ & 2.99 & $\mathrm{Ma}$ & 2.94 & $\mathrm{Ma}$ & 2.97 & Ma \\
\hline 13. Aktibo sa sistemang bayanihan o pakikipagtulungan & 3.00 & $\mathrm{Ma}$ & 2.88 & $\mathrm{Ma}$ & 2.94 & $\mathrm{Ma}$ \\
\hline 14. Marunong tumanaw ng utang na loob & 3.25 & $\mathrm{P}$ & 3.10 & $\mathrm{Ma}$ & 3.18 & $\mathrm{Ma}$ \\
\hline Kabuuang Mean & 3.14 & $\mathrm{Ma}$ & 3.02 & $\mathrm{Ma}$ & 3.08 & $\mathrm{Ma}$ \\
\hline
\end{tabular}

Maka-bansa - Ang talahanayan 3 ay naglalahad ng mga datos ukol sa dalas ng pagpapahalaga ng mga mag-aaral sa kaugaliang maka-bansa ayon sa kasarian. Batay sa mga datos, ang mga aytem na ang dalas ng pagpapahalaga ng mga respondente ayon sa kasarian ay "palagi” o pinapahalagahan sa lahat ng panahon ay "Iginagalang ang mga pambansang bayani" na may weighted mean na 3.45, "Ipinagmamalaki ang pagiging Pilipino", 3.44, "Iginagalang ang mga pambansang sagisag tulad ng pambansang awit, watawat ng Pilipinas at iba pa", 3.43, "Tinatangkilik ang pambansang wika sa pamamagitan ng paggamit nito sa tahanan o paaralan", 3.36, at "Sumasali sa flag raising ceremony", 3.32. Ito ay nangangahulugang patuloy na pinapahalagahan hanggang sa kasalukuyan ng mga respondente ayon sa kasarian ang mga sagisag ng bansang Pilipinas sa pamamagitan ng pagpapakita ng kanilang paggalang at pagtangkilik nito.

Sa kabuuang weighted mean na 3.25, ang dalas ng pagpapahalaga ng mga mag-aaral sa mga kaugaliang maka-bansa ayon sa kasarian ay palagi. Ibig sabihin, pinapahalagahan nila ang kaugaliang ito sa lahat ng panahon. Ayon sa pahayag ng Brainly.ph (2017), maipakita ng isang mamamayan ang kanyang pagiging maka-bansa, una, sa pamamagitan ng pagsunod sa mga batas, regulasyon, at alituntunin ng bansa upang maipakita ang pagmamahal at paggalang ng isang tao sa kanyang bansa o bayan. Pangalawa, sa pamamagitan ng "pagtangkilik sa sariling atin" mula sa mga lokal na magsasaka, manggugulay, mangingisda, at iba pa upang uunlad ang bayan at ang kapwa mamamayan. Pangatlo, sa pagmamahal at paggamit ng sariling wika dahil sumasalamin ito sa kultura at sa pagiging Pilipino ng isang tao, kaya mahalin, gamitin, ituro at ipasa ito sa mga susunod pang henerasyon. Ang pang-apat ay pagtatanggol sa bansa at hindi pagtalikod sa lupang sinilangan dahil ang paglilingkod, pagsisilbi, at pagrespeto nito ay isang magandang katangiang dapat taglayin ng isang mamamayang nagmamahal sa kanyang bayan.

Kaugnay nito, ibinahagi rin ni Plantilla (2021) ang pahayag ng isang Boy Scouts, na ang pagiging makabayan ay isinasapuso at hinihirang bilang isang likas na kaugaliang Pilipino at ipakita ito sa pamamagitan ng pakikisapi sa mga gawain ng pamahalaan, pangingialam sa mga pambansang suliranin at ipahayag ang ideya at pulso ng kabataan na magiging salamin at boses ng nakararami; pagkakaroon ng sapat na kaalaman sa mga batas na dapat sundin at gawin; pag-aaral ng Araling Panlipunan at ang pagkakaroon ng malawak na interes sa nakagisnang bansa kasama na ang mga pagpapahalagang kultural at responsibilidad ng bawat kabataan na ibida sa buong mundo kung ano ang Pilipino at kung ano ang Pilipinas. Umasa siya na maging daan sana ang globalisasyon hindi upang makalimutan ang sariling bayan, kundi mas ipagmalaki pa ang taglay na pagmamakabayan. 
Kaugaliang Pilipino at ang kabataan ngayon tungo sa programang edukasyon sa pagpapakatao

\section{Talahanayan 3}

Dalas ng pagpapahalaga ng mga respondente sa kaugaliang maka-bansa ayon sa kasarian

\begin{tabular}{|c|c|c|c|c|c|c|}
\hline \multirow{2}{*}{ Maka-bansa } & \multicolumn{2}{|c|}{ Babae } & \multicolumn{2}{|c|}{ Lalaki } & \multicolumn{2}{|c|}{ Kabuuan } \\
\hline & WM & Des & WM & Des & WM & Des \\
\hline 1. Sinusunod ang batas ng bansa & 3.20 & $\mathrm{Ma}$ & 3.27 & $\mathrm{P}$ & 3.24 & $\mathrm{Ma}$ \\
\hline 2. Tinatangkilik ang mga produkto ng bansa & 3.04 & Ма & 3.07 & $\mathrm{Ma}$ & 3.06 & $\mathrm{Ma}$ \\
\hline $\begin{array}{l}\text { 3. Iginagalang ang mga pambansang sagisag tulad ng } \\
\text { pambansang awit, watawat ng Pilipinas at iba pa }\end{array}$ & 3.50 & $\mathrm{P}$ & 3.35 & $\mathrm{P}$ & 3.43 & $\mathrm{P}$ \\
\hline 4. Sumasali sa flag raising ceremony & 3.38 & $\mathrm{P}$ & 3.26 & $\mathrm{P}$ & 3.32 & $\mathrm{P}$ \\
\hline $\begin{array}{l}\text { 5. Ninanais na magkaroon ng pagkakaisa ang lahat ng } \\
\text { Pilipino }\end{array}$ & 3.33 & $\mathrm{P}$ & 3.17 & $\mathrm{Ma}$ & 3.25 & $\mathrm{P}$ \\
\hline 6. Ninanais na mapanatili ang kapayapaang ng bansa & 3.29 & $\mathrm{P}$ & 3.21 & Мa & 3.25 & $\mathrm{P}$ \\
\hline 7. Mapagkawang-gawa & 2.97 & Мa & 3.05 & $\mathrm{Ma}$ & 3.01 & $\mathrm{Ma}$ \\
\hline 8. Hindi takot manindigan para sa katotohanan & 3.10 & Ма & 3.20 & $\mathrm{Ma}$ & 3.15 & $\mathrm{Ma}$ \\
\hline 9. Itinataguyod ang kabutihan ng nakararami & 3.15 & $\mathrm{Ma}$ & 3.07 & $\mathrm{Ma}$ & 3.11 & $\mathrm{Ma}$ \\
\hline 10. Iniisip ang kapakanan ng kapwa & 3.19 & Мa & 3.14 & $\mathrm{Ma}$ & 3.17 & $\mathrm{Ma}$ \\
\hline 11. Ipinagmamalaki ang pagiging Pilipino & 3.59 & $\mathrm{P}$ & 3.29 & $\mathrm{P}$ & 3.44 & $\mathrm{P}$ \\
\hline $\begin{array}{l}\text { 12. Tinatangkilik ang pambansang wika sa pamamagitan } \\
\text { ng paggamit nito sa tahanan o paaralan }\end{array}$ & 3.48 & $\mathrm{P}$ & 3.24 & $\mathrm{P}$ & 3.36 & $\mathrm{P}$ \\
\hline 13. Iginagalang ang mga pambansang bayani & 3.53 & $\mathrm{P}$ & 3.37 & $\mathrm{P}$ & 3.45 & $\mathrm{P}$ \\
\hline $\begin{array}{l}\text { 14. Ipinakikita ang pag-ibig sa bayan sa pamamagitan ng } \\
\text { pagtulong sa mga gawain ng komunidad o lipunan }\end{array}$ & 3.29 & $\mathrm{P}$ & 3.19 & $\mathrm{Ma}$ & 3.24 & $\mathrm{Ma}$ \\
\hline Kabuuang Mean & 3.29 & $\mathrm{P}$ & 3.20 & $\mathrm{Ma}$ & 3.25 & $\mathrm{P}$ \\
\hline
\end{tabular}

Maka-kalikasan - Ang talahanayan 4 ay naglalahad ng mga datos ukol sa dalas ng pagpapahalaga ng mga mag-aaral sa mga kaugaliang maka-kalikasan ayon sa kasarian. Batay sa mga datos, ang dalas ng pagpapahalagang "palagi" ay bukod tanging ang aytem 7 "Naglilinis ng paligid" na may weighted mean na 3.34 ng mga respondente ayon sa kasarian. Nangangahulugan ito na palaging pinapahalagan ng mga respondente ang paglilinis ng paligid. Samantala, ang karamihan sa mga aytem ay madalas nilang pinapahalagahan na sa kabuuan, ang mean ay 2.97 para sa mga babae at 2.96 naman sa mga lalaki. Ito ay nagpapakita na ang dalas ng pagpapahalaga ng mga respondente ayon sa kasarian ay madalas. Ibig sabihin, pinapahalagahan nila ito sa halos lahat ng panahon. Ang kinalabasang ito ay nangangahulugang hanggang sa kasalukuyan, madalas ang pagpapahalaga ng mga respondente sa kaugaliang nauukol sa pangangalaga sa kapaligiran o kalikasan. Ito ay nagpapahiwatig na may puso at pagkalinga ang kabataan sa inang kalikasan.

Sa pag-aaral na isinagawa ni Keh (2020), iminungkahi niya na nararapat na konektado ang pangangasiwa at ang ekolohiyang alituntuning sa katutubong paniniwala ng mga tao dahil ang kultura ay may mahalagang papel na ginagampanan para mabuo ang pagpapahalaga sa ekolohiya. Dagdag pa niya na kinakailangang gamitin ang kaalaman sa pagpapahalagang tradisyunal ng kultura sa paglutas ng suliraning ekolohikal sa mundo at ang kaalamang ito ay nararapat na saliksikin at gamitin nang mapagtanto ng tao ang halaga sa pangangalaga sa kalikasan. Pinagpasyahan niya na ngayon ang tamang panahon upang mabigyang-liwanang ang kahalagahan ng katatagan at pagkakaisa upang tugunan ang suliraning ekolohikal dahil ang pangangalaga sa kalikasan ay gawaing panlipunan sapagkat bawat isa ay bahagi sa isang tahanang panlahat.

\section{Talahanayan 4}

Dalas ng pagpapahalaga ng mga respondente sa kaugaliang maka-kalikasan ayon sa kasarian

\begin{tabular}{lcccccc}
\hline \multicolumn{1}{c}{ Maka-kalikasan } & \multicolumn{2}{c}{ Babae } & \multicolumn{2}{c}{ Lalaki } & \multicolumn{2}{c}{ Kabuuan } \\
& WM & Des & WM & Des & WM & Des \\
\hline 1. Itinatapon ang mga basura sa basurahan & 3.10 & $\mathrm{Ma}$ & 3.16 & $\mathrm{Ma}$ & 3.13 & $\mathrm{Ma}$ \\
2. Nagtatapon ng mga basura kahit saan & 2.10 & $\mathrm{Mi}$ & 2.37 & $\mathrm{Mi}$ & 2.34 & $\mathrm{Mi}$ \\
3. Inililibing ng maayos ang patay na hayop & 3.08 & $\mathrm{Ma}$ & 3.05 & $\mathrm{Ma}$ & 3.07 & $\mathrm{Ma}$ \\
$\begin{array}{l}\text { 4. Nagre-recycle ng mga magagamit pang basura tulad } \\
\text { ng plastic, lata, papel at bote }\end{array}$ & 2.65 & $\mathrm{Ma}$ & 2.87 & $\mathrm{Ma}$ & 2.76 & $\mathrm{Ma}$ \\
$\begin{array}{l}\text { 5. Ang mga nabubulok na basura ay inilagay sa } \\
\text { compost pit }\end{array}$ & 2.98 & $\mathrm{Ma}$ & 2.99 & $\mathrm{Ma}$ & 2.99 & $\mathrm{Ma}$ \\
$\begin{array}{l}\text { 6. Aktibo sa mga gawaing pangkalikasan tulad ng } \\
\text { paglilinis ng kapaligiran at pagtatanim ng mga } \\
\text { punongkahoy }\end{array}$ & 3.13 & $\mathrm{Ma}$ & 3.15 & $\mathrm{Ma}$ & 3.14 & $\mathrm{Ma}$ \\
\end{tabular}


Talahanayan 4 ...pagpapatuloy

\begin{tabular}{lcccccc}
\multicolumn{1}{c}{ Maka-kalikasan } & \multicolumn{2}{c}{ Babae } & \multicolumn{2}{c}{ Lalaki } & \multicolumn{2}{c}{ Kabuuan } \\
& WM & Des & WM & Des & WM & Des \\
\hline 7. Naglilinis ng paligid & 3.34 & $\mathrm{P}$ & 3.25 & $\mathrm{P}$ & 3.30 & $\mathrm{P}$ \\
8. Nagtatanim at nag-aalaga ng mga halaman & 3.04 & $\mathrm{Ma}$ & 3.07 & $\mathrm{Ma}$ & 3.06 & $\mathrm{Ma}$ \\
9. Nagtitipid ng kuryente at enerhiya & 2.93 & $\mathrm{Ma}$ & 2.94 & $\mathrm{Ma}$ & 2.94 & $\mathrm{Ma}$ \\
$\begin{array}{l}\text { 10. Hindi namumutol ng mga punong kahoy } \\
\text { 11. Hindi nanghuli ng mga ibon upang alagaan at }\end{array}$ & 3.19 & $\mathrm{Ma}$ & 2.85 & $\mathrm{Ma}$ & 3.02 & $\mathrm{Ma}$ \\
paglaruan & 3.07 & $\mathrm{Ma}$ & 2.84 & $\mathrm{Ma}$ & 2.96 & $\mathrm{Ma}$ \\
\hline Kabuuang Mean & & & & & & \\
\hline
\end{tabular}

\subsection{Kaibahan ng pagpapahalaga ng mga respondente}

Ang talahanayan 5 ay naglalahad ng kaibahan ng pagpapahalaga ng mga respondente sa kaugaliang Pilipino kung sila ay uuriin ayon sa kasarian. Batay sa resulta ng $t$-test, ang maka-Diyos ay may $t$-value na -0.02 at $p$-value na 0.311 , ang maka-kalikasan ay may $t$-value na -0.12 at $p$-value na 0.916 habang ang maka-bansa ay mayroong $t$-value na -1.39 at $p$-value na 0.165 . Ang tatlong kaugaliang nabanggit ay nagkakaroon ng $p$-value na mas mataas sa level of significance na 0.05 na dahilan ng pagkakaroon ng hindi makabuluhang interpretasyon. Ang ibig sabihin nito ay walang mahalagang kaibahan ang pagpapahalaga ng mga respondente sa tatlong Core Values. Sa kaugaliang maka-tao, ang $t$-value ay -2.42 at may $p$-value na 0.016 na mas mababa sa 0.05 lebel ng kabuluhan at lumabas na "Makabuluhan". Ibig sabihin magkakaiba ang pagpapahalaga ng mga babae at lalaki sa kaugaliang nauukol sa pagiging maka-tao.

Ang resultang ito ay nauugnay sa kinalabasan ng pag-aaral ni Perez (2012) na naghayag na ang pagkakaiba ayon sa kasarian ay natuklasan sa pang-araw-araw na karanasang ispiritwal, relasyon sa kani-kanilang ama, relasyon sa kauri, pagsasarili, positibong relasyon sa kapwa at layunin sa buhay habang wala namang natuklasang kaibahan sa aspekto ng positibo at negatibong epekto sa buhay, relasyon sa kani-kanilang ina at ng kanilang mga guro, kaalamang pangkalikasan, personal na pag-unlad at pagtanggap sa sarili.

\section{Talahanayan 5}

Kaibahan ng pagpapahalaga ng mga respondente sa kaugaliang Filipino kung uuriin ayon sa kasarian

\begin{tabular}{ccccccccc}
\hline Core Values & Kasari-an & $\mathrm{n}$ & $\mathrm{X}$ & $\mathrm{Sd}$ & $\mathrm{df}$ & $\mathrm{T}-$ Value & $\mathrm{p}$ & Interpretasyon \\
\hline Maka-Diyos & Lalaki & 164 & 2.77 & 0.55 & 389 & -1.02 & 0.311 & Hindi Makabuluhan \\
& Babae & 227 & 2.82 & 0.48 & & & & \\
Maka-Tao & Lalaki & 164 & 3.02 & 0.55 & 389 & -2.42 & 0.016 & Makabuluhan \\
& Babae & 227 & 3.14 & 0.48 & & & & Hindi Makabuluhan \\
Makabansa & Lalaki & 164 & 3.20 & 0.62 & 389 & -1.39 & 0.165 & \\
& Babae & 227 & 3.29 & 0.56 & & & & Hindi Makabuluhan \\
MakaKalikas & Lalaki & 164 & 2.96 & 0.54 & 389 & -0.12 & & \\
\hline
\end{tabular}

\subsection{Programa para sa edukasyong pagpapakatao}

Ang talahanayan 6 ay naglalaman ng programa para sa edukasyon sa pagpapakatao. Makikita sa talahanayan ang mga programang pang-edukasyon sa apat na Core Values. Inilantad dito ang iba't ibang layunin ng bawat programa nang lalong mapatibay ang paniniwala at pananalig ng mga mag-aaral sa Poong Maykapal, mapapahalagahan ng mga mag-aaral ang ugaling makatao, nalilinang ang mga kaugaliang maka-kalikasan, maisaisip at maisapuso ang pagpapahalaga sa mga kaugaliang pangmaka-bansa. 
Kaugaliang Pilipino at ang kabataan ngayon tungo sa programang edukasyon sa pagpapakatao

Talahanayan 6

Remedyal na programa para sa edukasyon sa pagpapakatao

\begin{tabular}{|c|c|c|c|c|c|c|c|}
\hline $\begin{array}{c}\text { Core } \\
\text { Values }\end{array}$ & Asignatura & Layunin & Proseso & Stratehiya & $\begin{array}{c}\text { Mga } \\
\text { Materyales }\end{array}$ & $\begin{array}{c}\text { Mga taong } \\
\text { gaganap }\end{array}$ & $\begin{array}{l}\text { Oras na } \\
\text { Ilalaan }\end{array}$ \\
\hline $\begin{array}{l}\text { Maka-ta } \\
\text { o }\end{array}$ & $\begin{array}{l}\text { Values, } \\
\text { MAPEH, } \\
\text { English, } \\
\text { Science, } \\
\text { Filipino, } \\
\text { Math }\end{array}$ & $\begin{array}{l}\text { Mas lalong } \\
\text { napapahalagah } \\
\text { an ng mga } \\
\text { mag-aaral ang } \\
\text { ugaling } \\
\text { maka-tao } \\
\end{array}$ & $\begin{array}{l}\text { Pagpapakita at } \\
\text { pagsasanib sa } \\
\text { kaugaliang makatao } \\
\text { sa lahat ng asignatura }\end{array}$ & $\begin{array}{l}\text { Pangkatang } \\
\text { gawain }\end{array}$ & $\begin{array}{l}\text { Mga } \\
\text { larawan }\end{array}$ & $\begin{array}{l}\text { Mga } \\
\text { Mag-aaral, } \\
\text { Mga guro, } \\
\text { Punong-Guro }\end{array}$ & $\begin{array}{l}5 \text { Oras bawat } \\
\text { lingo }\end{array}$ \\
\hline $\begin{array}{l}\text { Maka-Di } \\
\text { yos }\end{array}$ & $\begin{array}{l}\text { Values, } \\
\text { MAPEH, } \\
\text { English, } \\
\text { Science, } \\
\text { Filipino, } \\
\text { Math }\end{array}$ & $\begin{array}{l}\text { Napapatibay } \\
\text { ang } \\
\text { paniniwala, } \\
\text { pananalig ng } \\
\text { mga } \\
\text { mag-aaral sa } \\
\text { Diyos o Allah } \\
\text { at iba pa. }\end{array}$ & $\begin{array}{l}\text { Pagsasanib at } \\
\text { pagpapaalala sa } \\
\text { pagpapahalaga sa } \\
\text { ispirituwal na } \\
\text { paniniwala }\end{array}$ & $\begin{array}{l}\text { Pangkatang } \\
\text { gawain }\end{array}$ & $\begin{array}{l}\text { Mga } \\
\text { larawan }\end{array}$ & $\begin{array}{l}\text { Mga } \\
\text { Mag-aaral, } \\
\text { Mga guro, } \\
\text { Punong-Guro }\end{array}$ & $\begin{array}{l}2 \text { Oras bawat } \\
\text { lingo }\end{array}$ \\
\hline $\begin{array}{l}\text { Maka-ka } \\
\text { likasan }\end{array}$ & $\begin{array}{l}\text { Values, } \\
\text { MAPEH, } \\
\text { English, } \\
\text { Science, } \\
\text { Filipino, } \\
\text { Math }\end{array}$ & $\begin{array}{l}\text { Nalinang ang } \\
\text { mga } \\
\text { kaugaliang } \\
\text { maka-kalikasa } \\
\mathrm{n}\end{array}$ & $\begin{array}{l}\text { Pagsasanib sa } \\
\text { pagpapagawa sa mga } \\
\text { kaugaliang } \\
\text { maka-kalikasan }\end{array}$ & $\begin{array}{l}\text { Paglilinis ng } \\
\text { kapaligiran, } \\
\text { pagtatanim at } \\
\text { pag-aalaga ng } \\
\text { mga halaman }\end{array}$ & $\begin{array}{l}\text { Mga poster/ } \\
\text { larawan }\end{array}$ & $\begin{array}{l}\text { Mga } \\
\text { Mag-aaral, } \\
\text { Mga guro, } \\
\text { Punong-Guro }\end{array}$ & $\begin{array}{l}2 \text { Oras bawat } \\
\text { linggo }\end{array}$ \\
\hline $\begin{array}{l}\text { Maka-Ba } \\
\text { nsa }\end{array}$ & $\begin{array}{l}\text { Values, } \\
\text { MAPEH, } \\
\text { English, } \\
\text { Science, } \\
\text { Filipino, } \\
\text { Math }\end{array}$ & $\begin{array}{l}\text { Naisaisip at } \\
\text { naisapuso ang } \\
\text { pagpapahalaga } \\
\text { sa mga } \\
\text { kaugaliang } \\
\text { pang } \\
\text { maka-bansa }\end{array}$ & $\begin{array}{l}\text { Pagpapagawa sa } \\
\text { sampling mga } \\
\text { gawaing } \\
\text { pangmakabansa at } \\
\text { pagsasanib sa } \\
\text { pagtuturo sa lahat ng } \\
\text { asignatura }\end{array}$ & $\begin{array}{l}\text { Pangkatang } \\
\text { gawain }\end{array}$ & $\begin{array}{l}\text { Mga } \\
\text { Larawan ng } \\
\text { mga } \\
\text { Pambansan } \\
\text { g bayani }\end{array}$ & $\begin{array}{l}\text { Mga } \\
\text { Mag-aaral, } \\
\text { Mga guro, } \\
\text { Punong-Guro }\end{array}$ & $\begin{array}{l}5 \text { Oras bawat } \\
\text { linggo }\end{array}$ \\
\hline
\end{tabular}

\section{Konklusyon}

Ang dalas ng pagpapahalaga ng mga respondente ayon sa kasarian sa mga kaugaliang maka-Diyos, maka-tao at maka-kalikasan ay madalas habang sa pagiging maka-bansa ay palagi para sa mga babae at madalas naman para sa mga lalaki. Walang mahalagang kaibahan ang pagpapahalaga ng mga respondente kung sila ay uuriin ayon sa kasarian sa kaugaliang nauukol sa pagkamaka-Diyos, maka-kalikasan at maka-bansa ngunit sa pagiging maka-tao ay may mahalagang kaibahan.

\subsection{Rekomendasyon}

Batay sa mga natuklasan at konklusyon ng pag-aaral, ang mga sumusunod na rekomendasyon ay inihayag:

$>\quad$ Nararapat na palaging isasagawa ng mga guro ang pagsasanib ng edukasyon sa pagpapakatao sa lahat ng sabjek nang mapanatili ang pagpapahalaga ng kabataan sa mga mabubuting kaugaliang Pilipino.

$>\quad$ Nararapat na tulungan ng mga magulang ang kanilang mga anak na palaging pahalagahan ang mga kaugaliang Pilipino sa pamamagitan ng paggamit nito sa kanilang tahanan at pagsasanay nito sa araw-araw na pakikipamuhay.

$>\quad$ Higit na inirekomenda ang pagbibigay ng atensyon ng mga magulang at mga guro sa mga kabataan ukol sa paglinang ng kaugaliang maka-Diyos, maka-tao, at maka-kalikasan.

$>\quad$ Higit pang inirekomenda na ang ganitong pag-aaral ay isasagawa sa ibang institusyon upang mabatid kung ang ganitong kundisyon ay umiiral din.

\section{Sanggunian}

Alcober, S.A. (2016). Noon at ngayon: Kaugaliang Pilipino. Retrieved September 8, 2019, from http://shaneannalcober01.blogspot.com/2016/12/at-ngayonkaugaliang-pilipino.html 
Mansanadez, E.

Brainly.ph. (2017). Paano maipapakita ng isang mamamayan ang kanyang pagiging makabansa? Retrieved May 7, 2021, from https://brainly.ph/question/518167

Chico, M. L. M. (2012). Perception of Filipino values portrayed in Pinoy Big Brother. Online Journal of Communication and Media, 312-316.

Cordero, D. A., Jr. (2019). Moral decline in teens: The application of contextual theology. Philippine E-Journals Asia-Pacific Social Science Review, 13(2). Retrieved September 8, 2019, from https://ejournals.ph/article.php?id=9271

Credo, M. C., \& del Castillo, F. (2019). The effect of modeling approach in teaching catechesis vis-a-vis formation of value and prayer life of the Soup Kitchen Children in the Philippines. IJRSE, 8(4). https://doi.org/10.5861/ijrse.2019.4907

Cruz, C. (2015). Ugaling Pilipino: Noon at ngayon. Retrieved September 8, 2019, from https://magingalagadngsining.wordpress.com/2015/08/06/ugaling-pilipino-noon-at-ngayon/

del Castillo, F., del Castillo, C. D., Ching, G., Ackert, M., Aliño, M.A., \& Nob, R. (2021). Validation of the abrahamic forms of the centrality of religiosity scale (CRS-5, CRS-10, and CRS-15): Evidence from Selected University Students in the Philippines. Religions, 12, 84. https://doi.org/10.3390/rel12020084

Jumawan, R. O. (2019). The personal values of student assistants of St. Paul University Dumaguete. Philippine E-Journals, 7(2). Retrieved September 6, 2019, from https://ejournals.ph/article.php?id=10358

Kahambing, J. G. (2019). Understanding faith and the Ebed-Yahweh for Filipino values education. Journal of International Social Research, 12(65), 820-826. https://doi.org/10.17719/jisr.2019.3494

Keh, D. N. Jr. (2020). Stewardship of creation: Towards indigenous Filipino environmental principles. Social Ethics Society Journal of Applied Philosophy, 6(1), 91-115.

Llego, M. A. (2019). Policy guidelines in teaching Edukasyon sa Pagpapakatao (ESP). Teacherph: Professional Learning Online Community of Teachers and for Teachers. Retrievd September 6, 2019, from https://www.teacherph.com/edukasyon-sa-pagpapakatao/

McLeod, S. A. (2016). Albert Bandura's social learning theory. Simply Psychology. Retrieved September 6, 2019, from https://www.simplypsychology.org/bandura.html

MyInfo Basket.com (2020). Some ways to express Filipino nationalism. Retrieved September 6, 2019, from https://myinfobasket.com/some-ways-to-express-filipino-nationalism/

Obille, K, L. B. (2018). Information behaviour and Filipino values: An exploratory study. https://doi.org/10.1007/978-3-319-78105-1_57

Perez, J. A. (2012). Gender difference in psychological well-being among Filipino college student samples. International Journal of Humanities and Social Science, 2(13). Retrieved September 8, 2019, from http://www.ijhssnet.com/journals/Vol_2_No_13_July_2012/8.pdf

Plantilla, L. (2021). Ang pagmamahal sa Inang Bayan, ayon sa isang "boy scout". Philippine Information Agency. Retrieved May 7, 2021, from https://pia.gov.ph/features/articles/1023094

Reyes, J. (2015). Loób and kapwa: An introduction to a Filipino virtue ethics. An international Journal of the Philisophical Traditions of the East, 25(2). https://doi.org/10.1080/09552367.2015.1043173

Rosela, L. (2014). Pagkalimot sa mga tradisyong Pilipino. Retrieved September 6, 2019, from https://prezi.com/m3j263c5h4y7/pagkalimot-sa-mga-tradisyong-pilipino/

Ruto, J. J. (2017). Mga kaugalian at pagpapahalagang moral ng mga Pilipino. Retrieved September 6, 2019 , from https://prezi.com/yoankgyxlace/mga-kaugalian-at-pagpapahalagang-moral-ng-mga-pilipino-na-m/

Saito, I., Imamura, T., \& Miyagi, M. (2010). Filipino personality traits and values for social support: FOW as human resources for work life balance in Japan. Retrieved on May 7, 2021, from https://core.ac.uk/download/pdf/268585064.pdf

Suazo, R. (2019). Understanding the Filipino values of Filipino millennials on social media: A netnographic study. Retrieved September 7, 2019, from http://www.academia.edu/Documents/in/Filipino_Values

The Asian Parent Philippines. (n.d.). 11 na mabuting asal at kaugaliang Pilipino na dapat ituro sa mga anak. Retrieved January 15, 2020, from https://ph.theasianparent.com/kaugaliang-pilipino 